\title{
Propuesta pedagógica para el mejoramiento de la comprensión lectora, con base en la integración socioeducativa.
}

\section{Pedagogical proposal for the improvement of reading comprehension, based on socio-educational integration.}

\author{
Aydeé de las Mercedes Rodríguez-Martínez \\ Universidad Privada Norbert Wiener, Lima - Perú \\ ORCID: 0000-0002-4770-4166
}

Recibido: 27 de enero de 2021.

Aceptado: 14 de abril de 2021.

Publicado: 01 de mayo de 2021.

\begin{abstract}
Resumen - Para la sociedad actual es fundamental la correlación entre sociedad y educación, más aún si se toma en cuenta la influencia de las telecomunicaciones en los procesos humanos, lo cual ha reducido el contacto directo entre las personas. Asimismo, es un reto vigente de los procesos educativos en educación básica, el mejoramiento de las competencias lectoras, siendo el lenguaje una de las áreas primordiales en el desarrollo del ser humano en sociedad. En este contexto, se plantea el presente artículo con el objetivo de mostrar una propuesta pedagógica de integración socioeducativa para el mejoramiento de la comprensión lectora, denominada "PPECLE: Leer contigo, Leer conmigo", la cual fungió como estrategia pedagógica en el marco de una investigación cuantitativa con diseño cuasi experimental y nivel explicativo, en la cual se trabajó con 320 sujetos, entre estudiantes y padres del sexto grado de la Institución Educativa La Frontera, municipio Villa del Rosario, Colombia. Como técnica de recolección de información se utilizaron: un test de comprensión lectora (post-test) para evidenciar el desempeño luego de la propuesta, y cuestionarios para padres y estudiantes para medir el impacto de la propuesta. Respecto al mejoramiento de la comprensión lectora a través de la propuesta diseñada, se obtuvo a través de la prueba de Mann Whitney, la demostración de cambio con un alto nivel de significancia $(0,04)$ en el desempeño alcanzado respecto al pre-test que sirvió de base a la propuesta $\mathrm{Al}$ evaluar la eficacia de la propuesta como mecanismo pedagógico de integración socioeducativa para el trabajo lector, se encontraron resultados satisfactorios en los indicadores cumplimiento del objetivo (93\%), ambiente propiciado (83\%) y diseño (77\%). Se concluye que la propuesta mejora la comprensión lectora e impacta positivamente el ambiente familiar, escolar y social, además del estado emocional de los estudiantes.
\end{abstract}

Palabras clave: Propuesta pedagógica, comprensión lectora, integración socioeducativa.

\begin{abstract}
For today's society, the correlation between society and education is fundamental, even more so if the influence of telecommunications on human processes is taken into account, which has reduced direct contact between people. Likewise, it is a current challenge of the educational processes in basic education, the improvement of reading skills, with language being one of the primary areas in the development of human beings in society. In this context, this article is proposed with the aim of showing a pedagogical proposal of socioeducational integration for the improvement of reading comprehension, called "PPECLE: Read with you, Read with me", which served as a pedagogical strategy within the framework of a Quantitative research with a quasi-experimental design and explanatory level, in which 320 subjects were worked, among students and parents of the sixth grade of the La Frontera Educational Institution, Villa del Rosario municipality, Colombia. As a technique for collecting information, the following were used: a reading comprehension test (post-test) to demonstrate performance after the proposal, and questionnaires for parents and students to measure the impact of the proposal. Regarding the improvement of reading comprehension through the designed proposal, it was obtained through the Mann Whitney test, the demonstration of change with a high level of significance (0.04) in the performance achieved with respect to the pre-test that served as the basis for the proposal When evaluating the effectiveness of the proposal as a pedagogical mechanism of socio-educational integration for the reading work, satisfactory results were found in the indicators fulfillment of the objective (93\%), favorable environment (83\%) and design (77\% ). It is concluded that the proposal improves reading comprehension and positively impacts the family, school and social environment, as well as the emotional state of the students.

Keywords: Reading comprehension, socio-educational integration, pedagogical proposal.
\end{abstract}




\section{INTRODUCCIÓN}

Autores como [1], afirman que la interacción socioemocional incide favorablemente en los procesos lectores, lo cual avala la importancia de la participación de las familias y de la comunidad, en los procesos escolares, en el marco de una integración socioeducativa eficaz y necesaria [2]; se trata de un aprendizaje colaborativo entre actores educativos, en lo cual la familia juega un rol fundamental dado el acercamiento afectivo, presencial y continuo con el estudiante [3].

El diseño e implementación de una propuesta pedagógica para el mejoramiento de la comprensión lectora basada en la integración socioeducativa, fue uno de los objetivos planteados en el marco de la investigación doctoral denominada "Propuesta pedagógica de integración socioeducativa para el mejoramiento de la comprensión lectora en sexto grado, Institución Educativa La Frontera, 2019”. Esta investigación se formuló con el fin de dar respuesta al problema científico de cómo incorporar los factores socioemocionales, psicosociales y educativos en el quehacer pedagógico en torno a la lectura, partiendo de un problema percibido y evidenciado en la institución, en la cual se han reportado históricamente resultados de desempeño bajo en el área de lenguaje [4], y en el Índice Sintético de Calidad Educativa de dicha institución [5] [6].

A tal efecto, "PPECLE: Leer contigo, Leer conmigo", se consolida como una experiencia de investigación y pedagógica que pone el acento en los actores y el contexto tanto intra como extraescolar, en la cual son protagonistas los estudiantes y padres o cuidadores, vinculados con otros agentes sociales del contexto, y el docente como mediador o facilitador. La propuesta pedagógica se configura luego de una ardua revisión de antecedentes tanto nacionales como internacionales, del análisis del marco teórico existente sobre las distintas variables de investigación y del diagnóstico logrado, tanto de los estudiantes como de sus familias, el contexto escolar y el social.

\section{MARCO TEÓRICO}

Dentro del marco de la fundamentación teórica, se desarrollan dos apartados en los cuales se profundiza sobre proceso de la lectura y se especifican los campos de cada uno de sus niveles (literal, inferencial y crítico); y se plantea y argumenta la relación existente entre la integración socioeducativa y la comprensión lectora, como variables del estudio.

\section{a. Lectura y sus niveles}

La comprensión lectora es objeto de estudio de esta investigación como variable dependiente, en el sentido de que se pretende que la propuesta influya de manera positiva y significativa en los procesos y resultados cualitativos y cuantitativos de los estudiantes. La comprensión lectora es una competencia medular, no sólo en el desarrollo del lenguaje sino en todas y cada una de las áreas de formación. Según [7] la comprensión lectora es "la capacidad para entender lo que se lee, tanto en referencia al significado de las palabras que forman un texto, como con respecto a la comprensión global del texto mismo" (p.5). La lectura como el acto de comprender, de dar uso, reflexionar y relacionarse con textos escritos, para lograr objetivos propios, desarrollar el conocimiento, potenciarse a sí mismo y participar en sociedad [8].

Comprender un texto es escudriñar en los distintos elementos, factores y procesos que ello encierra. Para [9], la comprensión lectora implica procesos múltiples, unos simples y otros complejos, abordando desde la percepción y codificación o acceso léxico, hasta lo sintáctico y gramatical, las inferencias, la representación semántica y, finalmente, la comprensión. La comprensión lectora es un proceso de construcción de sentido, un activar las representaciones a partir de significados, es configurar una idea coherente y duradera a partir del texto y dentro de un contexto.

Los tres niveles de la lectura en la cual se basa la evaluación de estos procesos en pruebas tanto nacionales como internacionales, fueron definidos [10] a partir de las diversas formas de representación y expresión de la lengua: nivel literal, nivel inferencial y nivel de comprensión crítico. Algunos autores como [11], los denominan grados del desarrollo lector. En las pruebas TERCE estos niveles son tratados como procesos cognitivos [12]. Estos procesos son referidos por [13], como procesos mentales y estrategias cognitivas.

En torno al nivel literal, éste se entiende como la comprensión lectora que se logra a partir del conocimiento del significado de las palabras y de su función sintáctica. Ramírez ubica este nivel como de conocimiento del plano lingüístico [7]. El nivel literal es la lectura instrumental o decifrativa que se hace desde abajo, en la superficie del texto, en su parte lineal, el de las oraciones y proposiciones [14].

Respecto al nivel inferencial, [15] define inferir como deducir. Además de la formulación de hipótesis y deducciones, en este nivel se desentrañan las intenciones en los textos, sobrepasando lo que las palabras por sí solas expresan "y se interpreta haciendo uso de varios elementos del contexto, de la cultura y de los presaberes" (p.22) [16]. Al inferir, la persona que lee logra formar una representación mental a partir de la información textual disponible, conjugando, lo que está escrito de forma explícita y lo que está implícito o entre líneas, a través de procesos de interrelaciones entre los significados [17].

Para el nivel crítico textual, [18] precisa, que en este nivel lo fundamental son los cuestionamientos críticos; este proceso se caracteriza por la evaluación y reflexión de textos por los procesos de valoración y síntesis que incorpora [19]. En este nivel del lector emana la abducción creativa, en el sentido de que debe activar sus saberes para conjeturar, evaluar aquello que dice el texto e indagar por el modo como lo dice [20]. En la criticidad, se pone en relieve la intención ideológica de quien comunica algo a través del texto y las representaciones ideológicas de quien lee. Lipman enfatiza, que el pensamiento crítico es "sensible al contexto, orientado por criterios y lleva al juicio" (p. 115) [21].

Como se muestra en la Figura 1, los niveles de la comprensión lectora no se deben visualizar en gradación, no son escalones en ascenso. Cada uno se particulariza a partir de los procesos que encierra, pero se hallan en permanente correlación; al respecto, "la comprensión literal, la inferencial y la crítica intertextual son niveles de la lectura por los que un lector puede pasar, de manera indistinta, a medida que recorre un texto” (p. 23) [13]. Ningún nivel es mejor que otro, cada uno hace parte de un todo en el que la presencia y funcionalidad de uno y de otro están supeditadas. 


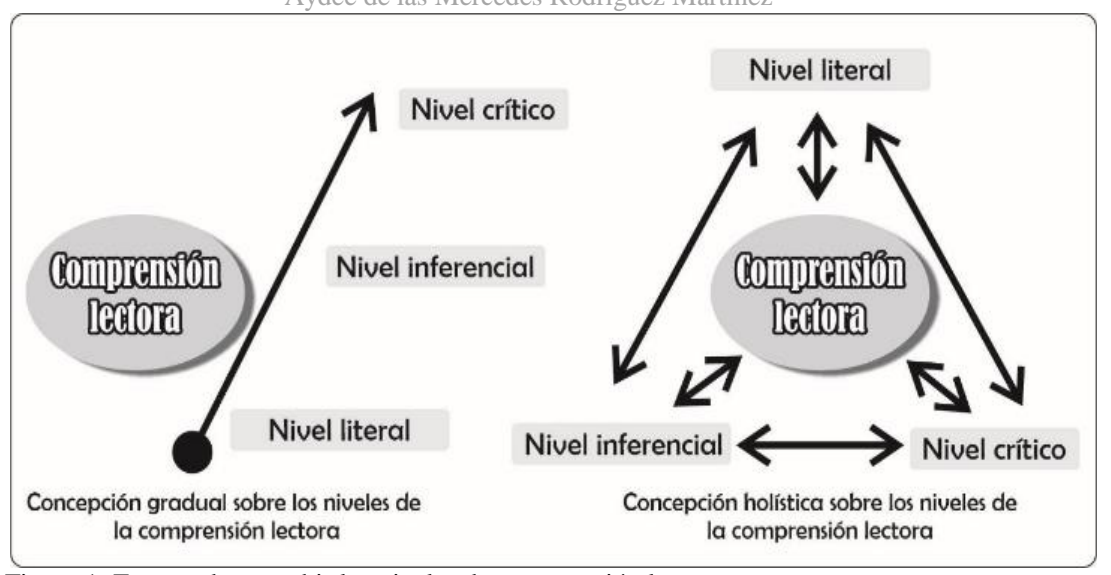

Figura 1: Formas de concebir los niveles de comprensión lectora.

Fuente: Elaboración propia.

\section{b. Relación entre la comprensión lectora y la integración socioeducativa}

El elemento filosófico y pedagógico que soporta la propuesta, es la incorporación del principio de integración social en la educación como fundamento potenciador del desempeño lector y en general, para el desarrollo de competencias del estudiante, tanto académicas, como personales y sociales. Teóricos como [22], [23] y [24], respaldan la imperativa incorporación del contexto social en los procesos de formación pedagógica, no sólo como la visualización de un diagnóstico, sino como la inclusión de sus agentes y procesos.

Según [25], los factores intersubjetivos, intrasubjetivos, contextuales y textuales son básicos y estructurales de la comprensión lectora. Para [26], [27] y [28], es clave la convergencia de ambos factores, pues son significativas y sólidas las repercusiones que proporciona la integración socioeducativa en los procesos de lectura. En palabras de [29], la lectura surge y se sostiene a partir de la consideración social y socializadora de la educación escolar. Por su parte, [27] reitera que el aprendizaje es significativo sólo si reconoce las necesidades e intereses de los estudiantes, es decir su individualidad y contexto.

Para [30], se manejan nueve propósitos de la lectura, entre ellos la obtención de información, el seguimiento de instrucciones, el aprendizaje, el placer, la comunicación, la lectura en voz alta y la comprensión. En concordancia [31], acentúa la importancia de la lectura dialógica entendida como un proceso intersubjetivo en el que cada persona reflexiona críticamente sobre el texto y el contexto dentro de su ser tanto social como individual. Reitera [19] que, en la lectura, se deben tener en cuenta tres tipos de contexto: el textual, representado por las relaciones que establece un enunciado con otros enunciados que lo rodean en el mismo texto; el extratextual, que abarca aspectos como el clima, el espacio físico o la posición del lector al leer; y, el psicológico, que se enfoca en el estado anímico del lector en el momento de leer el texto.

Por otra parte, el enfoque semántico comunicativo prioriza la construcción de significados por parte del propio sujeto y asume la naturaleza social del lenguaje; este enfoque pone el acento en la significación que integra a las demás competencias de la lengua (sintáctica, semántica y pragmática). Para [32], en los procesos de lectura desde la mirada semántica comunicativa se reconoce al estudiante como gestor de los propios saberes, se destaca el sentido del conflicto, se fomenta la cooperación y el encuentro, se impulsan actividades prácticas que permiten analizar e incorporar las características del mundo social y las condiciones del contexto, se propicia la cognición y metacognición, se fortalece la evaluación para definir logros y oportunidades de mejoramiento, se aprovecha la riqueza de los ambientes diversos, el uso de recursos auténticos y funcionales acordes con el contexto y con los propósitos de cada sesión y se plantean estrategias orientadas a la discusión, argumentación y socialización de experiencias, desde diferentes perspectivas y en un ambiente de respeto y tolerancia.

Según [33], un lector competente debe ir más allá de la parte gramatical, para comprender el contenido que subyace al texto y siendo consciente del contexto que lo suscitó, de los usos lingüísticos que el autor introdujo, del encuentro ideológico que se produce por el encuentro de esos mundos; la lengua como instrumento primordial para el desarrollo del hombre hace parte de su cultura, de su diario vivir y con ella se introducen subjetividades, modos de ser, sentir, pensar y actuar que se encuentran cuando se propicia la lectura (autor - lector - culturas).

\section{METODOLOGÍA O PROCEDIMIENTO}

La investigación en la cual se fundamenta la propuesta utilizada como estrategia pedagógica, fue de enfoque cuantitativo, con diseño cuasi experimental y nivel explicativo [34], en la cual se trabajó con 320 sujetos, 160 estudiantes y 160 padres del sexto grado de la Institución Educativa La Frontera, municipio Villa del Rosario, Colombia. Para los estudiantes, se trabajó con un grupo experimental y uno de control, cada uno de éstos compuesto por 80 sujetos. Como técnica de recolección de información se utilizó la encuesta, siendo los instrumentos un test de comprensión lectora post, y un cuestionario de integración socioeducativa para padres y otro para estudiantes. A tal efecto, se trató de un proceso metódico en cuatro etapas: diseño de la propuesta; implementación de la propuesta; medición del desempeño final con el post-test; medición del impacto de la propuesta.

Para la medición de comprensión lectora, se utilizó el instrumento validado de [35]. Esta prueba se constituye de 12 ítems de selección simple, 4 por cada dimensión (nivel literal, inferencial y crítico), sobre la base de preguntas alusivas a un texto. Para efectos de análisis e interpretación se hizo uso de la escala de desempeño establecida en Colombia por [4], la cual se distribuye en cuatro niveles: avanzado, satisfactorio, mínimo e insuficiente. A este instrumento se le calculó la confiabilidad con Alpha de Cronbach [34], encontrándose un índice de 0.81. En el proceso de medición del impacto de la propuesta, se diseñaron y aplicaron dos cuestionarios validados por expertos, cada uno con 5 ítem representativos de los siguientes criterios: cumplimiento del objetivo general, diseño y actividades, clima o ambiente de las sesiones, 
compromiso y cumplimiento con acciones y tareas y, uso de recursos, tanto físicos como humanos. Se introdujo una escala de valoración con tres descriptores: insuficiente ( 1 punto), aceptable ( 2 puntos) y satisfactorio ( 3 puntos).

A efectos del procesamiento y análisis de datos, se utilizó la estadística descriptiva para la tabulación de resultados y cálculo de proporciones del post-test y de los cuestionarios de impacto; por su parte, la estadística inferencial a través de pruebas de normalidad y correlaciones en el proceso de medición del impacto de la propuesta, con apoyo en el paquete estadístico SPSS.

\section{RESULTADOS ANÁLISIS E INTERPRETACIÓN}

\section{a. Caracterización de la propuesta}

Una propuesta pedagógica no puede mirarse desde un enfoque sólo instrumental; ésta se constituye en un mecanismo diseñado para facilitar la formación y la información cultural, considerando las características del sujeto que aprende, la disciplina que se enseña y el contexto [36]. Tampoco puede reducirse a las paredes del recinto escolar ni minimizarse en una relación entre dos (estudiante y docente); hoy más que nunca, los procesos escolares han tomado vigor en el hogar y en otros contextos, con más actores, con una gran diversidad de medios y herramientas y con nuevos retos, competencias y contenidos [37].

El sistema de acciones pedagógicas consolidado en la investigación, parte de un reconocimiento y diagnóstico de los sujetos y del contexto desarrollado por [38], en el cual se determinó que el nivel con más baja valoración de desempeño fue el crítico-intertextual con 48,44 aciertos; el nivel literal alcanzó el 65,31\%, mientras que el nivel inferencial obtuvo 65,63\% de aciertos respectivamente, lo cual revela que la mayoría de los estudiantes se encuentran en un desempeño mínimo y satisfactorio. Con base en estos resultados, la propuesta se fundamenta en la lectura desde sus niveles literal, inferencial y crítico textual, y consta de con fundamentos y un marco axiológico, según se muestra en la Figura 2.

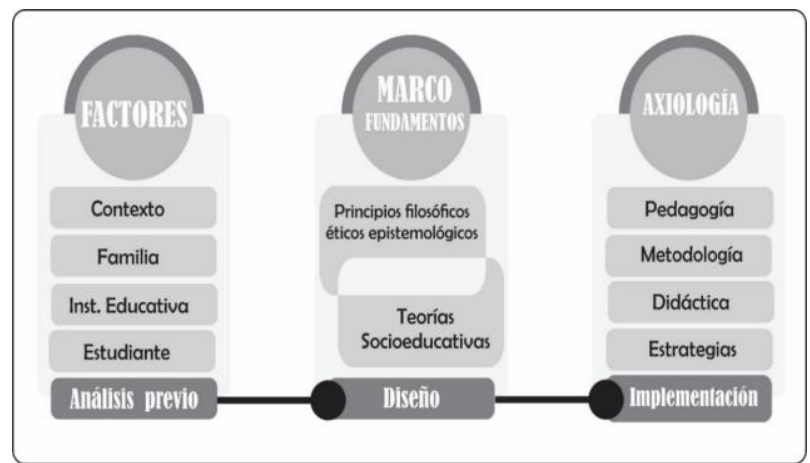

Figura 2: Estructura de una propuesta pedagógica.

Fuente: Elaboración propia.

En la referida figura se presentan factores de entrada en la integración socioeducativa, constituidos por el contexto, la familia, la institución y el estudiante, los cuales se enlazan desde principios filosóficos de educación y la sociología, requiriéndose un marco integrador propositivo con elementos pedagógicos, metodológicos, didácticos y estratégicos, los cuales sustenten el diseño de la propuesta u su implementación. Para su logro, fue necesario una lectura amplia y minuciosa de varios teóricos, de importantes organismos internacionales como el Fondo de las Naciones Unidas para la Infancia (UNICEF) [39], la Organización de las Naciones Unidas para la Educación, la Ciencia y la Cultura (UNESCO) [40], la Organización para la Cooperación y el Desarrollo Económicos (OCDE) [41], y nacionales como el Instituto Colombiano para la Evaluación de la Educación (ICFES) [4], además de los lineamientos y orientaciones emanadas por el Ministerio de Educación Nacional (MEN) [42] [43], a través de cartillas, manuales, proyectos o programas.

La propuesta está conformada por 30 sesiones alrededor de la lectura y sobre la base de la integración familiar, pero con procesos transversales como el lúdico, artístico, ético, de competencia ciudadana y tecnológicos, entre otros, según se muestra en la Tabla 1, abordando el proceso de sensibilización y socialización y el balance y evaluación de la experiencia a través de la propuesta.

Tabla 1: Naturaleza de las actividades.

\begin{tabular}{|c|c|}
\hline Nombre de la actividad & Descripción \\
\hline $\begin{array}{c}\text { Navego entre personajes } \\
\text { por el alfabeto }\end{array}$ & $\begin{array}{c}\text { La actividad apunta a reconocer en la voz de los estudiantes sus experiencias lectoras, su gusto y } \\
\text { comprensión por el mundo literario. Se trata de la presentación de un personaje siguiendo el orden } \\
\text { del alfabeto, seguido por un comentario o referencia }\end{array}$ \\
\hline $\begin{array}{c}\text { Sazonando el caldo de } \\
\text { cultivo }\end{array}$ & $\begin{array}{c}\text { Se pretende conocer la biblioteca familiar, escolar, municipal o digital y adecuar un rincón de } \\
\text { lectura en el hogar y en el espacio escolar }\end{array}$ \\
\hline $\begin{array}{c}\text { Los favoritos de nuestros } \\
\text { padres }\end{array}$ & $\begin{array}{c}\text { Busca que los padres se muestren como sujetos lectores ante sus hijos de manera que sean un } \\
\text { ejemplo y una fuerte motivación para sus nuevas experiencias de lectura. Facilita la conformación de } \\
\text { red de lectores para compartir los libros y sus contenidos }\end{array}$ \\
\hline Nuestros favoritos & $\begin{array}{c}\text { Incita al respeto y reconocimiento a la elección personal del lector, a su subjetividad y gusto } \\
\text { personal; incluye un intercambio de favoritos entre estudiantes }\end{array}$ \\
\hline Narraciones & $\begin{array}{c}\text { Tu lees, yo te escucho: Entre iguales. En el hogar, padres e hijos. Entre el estudiante y el docente } \\
\text { facilitador }\end{array}$ \\
\cline { 2 - 2 } & $\begin{array}{c}\text { Yo leo, tú me escuchas: Entre iguales. En el hogar, padres e hijos. Entre el estudiante y el } \\
\text { docente facilitador }\end{array}$ \\
\hline
\end{tabular}




\begin{tabular}{|c|c|}
\hline Tendedero de fábulas & $\begin{array}{l}\text { Se exponen a manera de galería fabulas impresas con el fin de tomarlas y leer de manera libre y } \\
\text { espontánea. Estos tipos de textos por su estructura e intención comunicativa, son altamente propicios } \\
\text { para la reflexión, el debate y la valoración }\end{array}$ \\
\hline Leyendo con autores & $\begin{array}{l}\text { El encuentro entre autores y lectores, además de permitir un acercamiento personal y más } \\
\text { completo sobre la obra literaria, es un ambiente propicio para el aprendizaje social y afectivo. Acerca } \\
\text { a los agentes de la lectura y ambienta ese encuentro ideológico que ella suscita }\end{array}$ \\
\hline Cuentos viajeros & $\begin{array}{l}\text { Permite y promueve la circulación de los libros, por medio del préstamo o el intercambio, entre } \\
\text { personas o con la Institución }\end{array}$ \\
\hline $\begin{array}{l}\text { Velada por la tradición } \\
\text { oral }\end{array}$ & $\begin{array}{l}\text { Compartir leyendas y mitos leídos en encuentros generacionales, motivando un conversatorio } \\
\text { alrededor de estos relatos en ambientes simulados }\end{array}$ \\
\hline Cuento en pantalla & Lectura de libros digitales o en formatos como diapositivas o videos \\
\hline $\begin{array}{l}\text { Alrededor de un } \\
\text { audiolibro }\end{array}$ & $\begin{array}{l}\text { Reconocer y asumir el estilo de lectura oral además de la visual a partir de la diferenciación } \\
\text { misma de los estilos de aprendizaje }\end{array}$ \\
\hline Un cuentero/a nos visita & $\begin{array}{l}\text { Extensión de la invitación y recibimiento de cuenteros de la zona por medio de cualquier canal de } \\
\text { comunicación: presencial, virtual }\end{array}$ \\
\hline $\begin{array}{c}\text { Reconozco los } \\
\text { Booktubers y Bookstrailers }\end{array}$ & Además, es importante la lectura de una sugerencia hallada \\
\hline Lectura entre iguales & Lectura compartida y en voz alta \\
\hline $\begin{array}{l}\text { Feria de la narración } \\
\text { literaria }\end{array}$ & $\begin{array}{l}\text { Compartir con otras personas, amigos, compañeros o familiares sus acercamientos a los libros y } \\
\text { procesos de lectura, promoviendo la lectura y valorando sus propios gustos, procesos y avances }\end{array}$ \\
\hline Mis lecturas son mis alas & $\begin{array}{l}\text { En unas alas diseñadas con cartón, los estudiantes exponen todos los libros leídos a través de la } \\
\text { escritura de títulos, frases o dibujos alusivos }\end{array}$ \\
\hline
\end{tabular}

Fuente: Elaboración propia.

Esta propuesta se elaboró y diseñó dentro de la pedagogía del aprendizaje significativo y el enfoque semántico comunicativo que pondera la construcción de significados por parte del sujeto; así mismo, asume la naturaleza social del lenguaje. De acuerdo con [44], la lectura como proceso pedagógico, se concretiza en la capacidad del docente para insertar al estudiante en la selección, comprensión y discusión de textos dentro de contextos significativos. [45] considera que el proceso de lectura no se desarrolla de forma espontánea, sino que hay que enseñarlo poco a poco y se aprende, a partir de objetivos concretos y en contextos significativos de lectura. Para la enseñanza de la lengua y dentro de ella, de la lectura, es fundamental el proceso de socialización, ya que ésta "se aprende desde la interacción, en la necesidad del uso, en la práctica y en la participación en contextos auténticos” (p.4) [16].

Cada sesión de la propuesta, se identifica por su número y título. En cada una de ellas se especifican los actores para quienes va dirigida, entendiendo que el rol del docente es el de facilitador. Al organizar cada sesión, se ha formulado el propósito y el objetivo de cada una, el nivel de lectura que involucra la propuesta pedagógica contenida y los estándares y derechos básicos de aprendizaje que tienen lugar a partir de las actividades. Algunas sesiones son en el ambiente escolar y otras en el hogar. Para orientar la implementación se detalla el modo en que se plantea el inicio, desarrollo y cierre de cada sesión. Al final se da cuenta de los recursos y de la duración aproximada.

Como se observa en la Tabla 1, PPECLE retoma las estrategias para la comprensión que organiza [46], en tres momentos, antes, durante y después de la lectura, definiendo para cada uno de ellos, estrategias pedagógicas específicas. Para el primer momento, antes de leer, se busca reconocer los objetivos concretos de la lectura y despertar los conocimientos previos relevantes; luego, durante la lectura, se pretende propiciar inferencias, revisar y comprobar la comprensión lograda, de modo que se permita enmendar errores y superar dificultades, en caso de ser necesario; finalmente, después de la lectura, es el momento para identificar las ideas fundamentales del texto, sintetizar, ampliar, valorar, contrastar y complementar. Llevar a cabo cada etapa con entusiasmo y asertividad garantiza el gusto y la construcción de sentido.

La propuesta pedagógica formulada incluye textos tanto continuos como discontinuos, pero todos narrativos y literarios. Esta decisión se basa en el hecho de que este tipo de textos permite una lectura más estética o connotativa. Los textos narrativos regalan una experiencia cognitiva y placentera, son una puerta abierta para la afirmación del yo, para el encuentro con el otro y lo otro, un estímulo para la imaginación, un reconocimiento de la diversidad, una guarda de la memoria, un avivar de la identidad y la cultura, un mecanismo eficaz para la construcción de la buena ciudadanía. Junto a ello, la propuesta perfilada, da apertura a distintos tipos de lectura (silenciosa, en voz alta, solitaria, compartida, entre otras) e incorpora el uso de las Tecnologías de la Información y las Comunicaciones (TIC), y de otros recursos como los Centro de Recursos para el Aprendizaje (CRA) [47]. Asimismo, permite la participación de otros miembros de la sociedad como los cuenteros o escritores, fortaleciendo la difusión y conservación de relatos propios de la tradición oral, fuente de identidad de los pueblos [30].

"PPECLE: Leer contigo, leer conmigo", asume la lectura como un conjunto de habilidades que se entrecruzan permanentemente (leer, escribir, escuchar y hablar) y que incluye diversos procesos, simples y complejos. [48] resalta el proceso interactivo de la lectura en cuanto a la construcción de sentido que hace el lector del texto y el proceso transaccional, puesto que el lector y el texto son codependientes. La lectura es también un proceso de integración en el que otras habilidades y procesos tienen lugar y se constituye en un proceso de transformación, pues a partir de ella, se generan cambios en distintas direcciones y agentes.

La lectura es también un punto focal para la formación en valores y emociones. La teoría de la motivación humana establecidas por [49], resalta que el amor, la participación y la autoestima, son necesidades fundamentales para el desarrollo del ser, de su realización, conocimiento y comprensión. PPECLE, además, busca aumentar la motivación y autoestima de los estudiantes al ser escuchados, reconocidos, orientados. Cuando el estado emocional y afectivo es positivo, con ese ese logro se fortalecen igualmente las relaciones con el otro y se amplían los alcances en otras competencias.

Para [50], la lectura es un derecho vital, un proceso fundamental para la formación de la subjetividad y el desarrollo del ser social; la lectura le permite al ser humano descubrirse y construirse, evocar su propia voz, lo motiva a sentir el poder que da la palabra, lo levanta en momentos de adversidad, le impulsa a dibujar sus sueños y aspiraciones, sus miedos y flaquezas, le da libertad y evidencia la libertad del otro. La propuesta "PPECLE: Leer contigo, leer conmigo", se constituye en una oportunidad y un espacio propicio para ejercer ese derecho vital de la lectura y es 
una ocasión para el encuentro del estudiante consigo mismo, con sus iguales, con sus docentes, con su familia, con su comunidad. Incluye estrategias tanto formales como lúdicas que les permiten a los estudiantes desarrollar sus otras competencias, además de cognitivas, emocionales, afectivas y sociales.

En el diseño de la propuesta se incorporaron aspectos muy significativos de [43], además del material dirigido a docentes y bibliotecarios escolares, para orientar la vinculación de los padres de familia en el acompañamiento a los procesos de aprendizaje de la lectura y la escritura de sus hijos, a partir de actividades lúdicas y sencillas que ponderan la comunicación y la afectividad [51]. También se incorporaron los fundamentos del programa LECXIT [52], el cual pretende incrementar el éxito educativo de los niños y niñas a través del trabajo de las escuelas, las familias y el voluntariado, con base en la lúdica [53].

\section{b. Medición de la eficacia (impacto) de la propuesta}

La eficacia de la propuesta se evidencia en los resultados obtenidos al aplicar el test de comprensión de lectura después de la implementación, y compararlos con los resultados del pre-test que sirvieron de base a la propuesta. En primer lugar, se aplicó la prueba de normalidad de datos Kolmogorov-Smirnov [34], determinándose que se trata de una muestra que no tiene comportamiento normal al obtenerse un $\mathrm{p}=0,036<\alpha=$ 0,05. Se pasó a determinar la variación entre los datos del pre-test y el post-test con la prueba U de Mann Whitney [34], cuyos resultados se muestran en la Tabla 2. Al respecto, el índice del nivel literal fue de 0,273 y en el nivel inferencial 0,48 , ambos $>\alpha=0,05$, concluyéndose que no hay significancia; en el nivel crítico textual se obtuvo 0,040 y en la comprensión lectora general se obtuvo 0,004 , concluyéndose en muy alta significancia con $\mathrm{p}<\alpha=0,05$. Se concluye que el carácter dinámico y dialógico de la propuesta, además de su diversidad de recursos, espacios y estrategias, fortalece los niveles complejos de la inferencia y la crítica en la comprensión de textos.

Tabla 2: Prueba U de Mann-Whitney.
\begin{tabular}{|c|c|c|}
\hline \multirow{2}{*}{} & Pretest & Postest \\
\cline { 2 - 3 } & Nivel Literal & Nivel Literal \\
\hline U de Mann-Whitney & 3033,000 & 2891,500 \\
\hline W de Wilcoxon & 6273,000 & 6131,500 \\
\hline Z &,- 591 & $-1,096$ \\
\hline Sig. asintót. (bilateral) &, 555 &, 273 \\
\hline & Nivel Inferencial & Nivel Inferencial \\
\hline U de Mann-Whitney & 3168,000 & 2650,500 \\
\hline W de Wilcoxon & 6408,000 & 5890,500 \\
\hline Z &,- 113 & $-1,979$ \\
\hline Sig. asintót. (bilateral) &, 910 &, 048 \\
\hline & Nivel Crítico & Nivel Crítico \\
\hline U de Mann-Whitney & 3045,000 & 2624,500 \\
\hline W de Wilcoxon & 6285,000 & 5864,500 \\
\hline Z &,- 551 & $-2,051$ \\
\hline Sig. asintót. (bilateral) &, 581 &, 040 \\
\hline & N. Comp. & N. Comp. \\
\hline U de Mann-Whitney & 3174,500 & Lectora \\
\hline W de Wilcoxon & 6414,500 & 2355,000 \\
\hline Z &,- 088 & 5595,000 \\
\hline Sig. asintót. (bilateral) &, 930 & $-2,917$ \\
\hline
\end{tabular}

Fuente: Elaboración propia.

Posteriormente, los padres y estudiantes evaluaron la propuesta de intervención en la última sesión, cuyos resultados se muestran en la Tabla 3. La estadística descriptiva muestra que la propuesta posee una importante fortaleza en el indicador de cumplimiento del objetivo, con un porcentaje de satisfacción de 93\%. Valoración altamente positiva obtuvo el clima o ambiente (83\%) junto con el diseño (77\%). Las oportunidades de mejoramiento se hallan especialmente en los criterios de compromiso y cumplimiento con las actividades y tareas con un porcentaje de $62 \%$ y uso de recursos $50 \%$.

Tabla 3: Evaluación de la eficacia de la propuesta por parte de padres y estudiantes.

\begin{tabular}{|c|c|c|c|c|c|c|c|c|c|c|}
\hline & \multicolumn{2}{|c|}{$\begin{array}{l}\text { Cumplimiento } \\
\text { del objetivo }\end{array}$} & \multicolumn{2}{|c|}{$\begin{array}{c}\text { Diseño; } \\
\text { Actividades }\end{array}$} & \multicolumn{2}{|c|}{$\begin{array}{c}\text { Clima } \\
\text { o ambiente }\end{array}$} & \multicolumn{2}{|c|}{$\begin{array}{l}\text { Compromiso, } \\
\text { cumplimiento }\end{array}$} & \multicolumn{2}{|c|}{$\begin{array}{l}\text { Uso de } \\
\text { recursos }\end{array}$} \\
\hline & Cant. & $\%$ & Cant. & $\%$ & Cant. & $\%$ & Cant. & $\%$ & Cant. & $\%$ \\
\hline Insuficiente & 0 & $0 \%$ & 6 & $8 \%$ & 3 & $4 \%$ & 12 & $15 \%$ & 12 & $15 \%$ \\
\hline Aceptable & 5 & $7 \%$ & 12 & $15 \%$ & 11 & $13 \%$ & 18 & $23 \%$ & 20 & $25 \%$ \\
\hline Satisfactorio & 75 & $93 \%$ & 62 & $77 \%$ & 66 & $83 \%$ & 50 & $62 \%$ & 48 & $60 \%$ \\
\hline
\end{tabular}

Fuente: Elaboración propia.

\section{CONCLUSIONES}

"PPECLE: Leer contigo, leer conmigo", tiene una mirada holística, ecléctica, constructiva del saber y comunicativa, es transversal a muchas áreas de formación y aprehende una variada rama de espacios, recursos y actividades; se aborda desde el enfoque hermenéutico que tiene su sustento en el encuentro múltiple y complejo que demanda la convergencia del fenómeno social y el educativo. Esta propuesta además de 
impactar en los procesos de lectura, contribuye en la consolidación de un mejor entorno familiar y social, atiende la personalidad de los estudiantes, sus emociones y gustos, su contexto mediato e inmediato y las personas que participan en sus procesos formativos y de crecimiento.

Los resultados evidencian que además de mejorar el nivel de comprensión lectora general, hubo un alto grado de satisfacción en la evaluación que de la misma hizo el grupo de estudiantes y padres o cuidadores que participó en las sesiones programadas. Ello demuestra que la integración social es fundamental en los procesos de comprensión lectora. La mejora que se aprecia en el proceso de comprensión lectora luego de la intervención pedagógica, evidencia que acentuar el factor socioeducativo, potencia el nivel inferencial y crítico textual referentes a la macroestructura y la interpretación. El aspecto dialógico de la propuesta, su ajuste al contexto inmediato y mediato del estudiante, además del corte lúdico y de reconocimiento de las emociones, constituyen gran parte del acierto.

La escuela debe abrir sus puertas y fortalecer su trabajo de proyección y participación con la comunidad, tanto en presencialidad como virtualidad, incorporando a las familias desde un rol protagónico y reconociendo las oportunidades que el texto y el contexto le ofrece.

\section{REFERENCIAS}

[1] F. Marmolejo y A. Jiménez, "Inferencias, modelos de situaciones y emociones en textos narrativos. El caso de los niños en edad preescolar". Revista Intercontinental de Psicología y Educación, vol. 8, n² 2, pp. 93-138, 2006.

[2] L. Alvarado y M. García, "Características más relevantes del paradigma socio-crítico: su aplicación en investigaciones de educación ambiental y de enseñanza de las ciencias realizadas en el Doctorado de Educación del Instituto Pedagógico de Caracas". Sapiens. Revista Universitaria de Investigación, vol. 9, $\mathrm{n}^{\circ}$ 2, pp. 187-202, 2008.

[3] L. Bonell, "La promoción de la participación educativa de las familias en la escuela desde una perspectiva transformadora: un estudio de casos", [Tesis Doctoral], España: Universidad de Valladolid, 2016.

[4] Instituto Colombiano para la Educación Superior, "SABER PRO Informe Nacional de Resultados 2016-2017". Bogotá: Ministerio de Educación Nacional, 2018.

[5] Ministerio de Educación Nacional, "Índice Sintético de Calidad Educativa de la Institución Educativa La Frontera”, 2017.

[6] Ministerio de Educación Nacional, "Índice Sintético de Calidad Educativa de la Institución Educativa La Frontera", 2018.

[7] L. Ramírez, "Desarrollo de competencias en la comprensión lectora: La comprensión lectora como una herramienta que apoya al desarrollo de nuevas competencias", España: Editorial Académica Española, 2017.

[8] Organization for Economic Cooperation and Development, "PISA for Development Assessment and Analytical Framework: Reading, Mathematics and Science", Paris: OECD Publishing, 2017.

[9] I. Escudero, "Las inferencias en la comprensión lectora: Una ventana hacia los procesos cognitivos en segundas lenguas". Revista Nebrija de Lingüística aplicada a la Enseñanza de Lenguas, vol. 4, n 7, pp 6-32, 2010. https://doi.org/10.26378/rnlael47127

[10] R. Strang, "Procesos del aprendizaje infantil", Buenos Aires: Paidós, 1965.

[11] M. Aravena. (2004). "Noción de inferencia y procesamiento inferencial en personas con daño cerebral”. Onomázein. [PDF]. 10 (2), pp 145-162. Disponible en [http://www.redalyc.org/pdf/1345/134517755007.pdf

[12] Organización de las Naciones Unidas para la Educación, la Ciencia y la Cultura, "Tercer Estudio Regional Comparativo y Explicativo (TERCE). Análisis curricular", Santiago de Chile: Oficina Regional de Educación para América Latina y el Caribe (OREALC/UNESCO), 2013.

[13] A. Montero, L. Zambrano y C. Zerpa, “La comprensión lectora desde el constructivismo”. Cuadernos Latinoamericanos, vol. 25, n 1 , pp. 9-27, 2014.

[14] L. Tuffanelli, "Didácticas de Las Operaciones Mentales”, Madrid: Ediciones Narcea S.A., 2010.

[15] Real Academia Española, "Inferir", 2010.

[16] Ministerio de Educación Nacional, "Lineamientos Curriculares en Lengua Castellana”, Bogotá, 1998.

[17] G. Parodi, "Comprensión de textos escritos". Argentina: Universidad de Buenos Aires, 2005.

[18] M. Rodríguez, "Estrategias didácticas para la comprensión lectora en estudiantes de segundo grado de la básica primaria". Gestión Competitividad e Innovación, vol. 4, n² 2, pp. 1-14, 2016.

[19] J. Martín, "La comprensión de textos expositivos en el aula de ciencias sociales en la educación secundaria", [Tesis Doctoral], España: Universidad de Granada, 2013.

[20] U. Eco, "Los límites de la interpretación”, España: Editorial De Bolsillo, 1992.

[21] M. Lipman, "Pensamiento complejo y educación”, Madrid: Ediciones de La Torre, 1997.

[22] R. Thorndike, "La comprensión lectora: Educación en quince países”, Nueva York: John Wiley, 1973.

[23] C. Argyris, D. Schon, "Organizational Learning: A Theory of Action Perspective”, San Francisco: Addison Wesley, 1978.

[24] D. Ausubel, "Psicología educativa. Un punto de vista cognoscitivo", México: Trillas, 1995.

[25] G. Marciales, "Pensamiento crítico: diferencias en estudiantes universitarios en el tipo de creencias, estrategias e inferencias en la lectura crítica de textos", [Tesis Doctoral], Madrid: Universidad Complutense de Madrid, 2003.

[26] F. Marmolejo y A. Jiménez, "Inferencias, modelos de situaciones y emociones en textos narrativos. El caso de los niños en edad preescolar". Revista Intercontinental de Psicología y Educación, vol. 8, n² 2, pp 93-138, 2006.

[27] D. Saldaña, "Teoría de la mente y lectura en las personas con trastornos del espectro autista: hipótesis para una relación compleja". Revista de Logopedia, Foniatría y Audiología, vol. 28, n² 2, pp. 117-125, 2008. https://doi.org/10.1016/S0214-4603(08)70050-0

[28] R. Cárdenas y E. Santrich, "Factores asociados a la compresión lectora en los estudiantes de noveno grado de la I.E.D. "Jesús Maestro Fe y Alegría" de Barranquilla", [Tesis de Maestría], Barranquilla: Universidad de la Costa, 2015.

[29] C. Coll y I. Solé, "Los profesores y la concepción constructivista”, Barcelona: Graó, 1993.

[30] I. Solé, "Estrategias de comprensión de la lectura", Barcelona: Graó,1996.

[31] R. Valls, M. Soler y R. Flecha, "Lectura dialógica: Interacciones que mejoran y aceleran la lectura". Revista Iberoamericana de Educación, vol. 46, n' 1 , pp. 71-87, 2008.

[32] M. Rodríguez y G. Jaimes, "El enfoque semántico comunicativo en la enseñanza de la lengua materna: Balance y perspectivas". Revista Universidad Distrital, vol. 4, n 1, pp. 9-13, 1999. https://doi.org/10.14483/22486798.2422

[33] M. Dubois, "El proceso de lectura, de la teoría a la práctica", 2015.

[34] R. Hernández, C. Fernández y P. Baptista, "Metodología de la investigación”, México: McGraw-Hill Interamericana, 2010. 
[35] M. Ochoa, "Martes de prueba sexto grado. Prueba de Lenguaje", 2018.

[36] J. Supo, "Cómo validar un instrumento", Perú: Biblioteca Nacional del Perú, 2013.

[37] H. Oviedo y A. Campos, “Aproximación al uso del coeficiente de Alfa de Cronbach”, Revista Colombiana de Psiquiatría, vol. 34, n 4, pp. 572-580, 2015.

[38] A. Rodríguez, "La integración socioeducativa, factor clave para una mejor comprensión lectora". INNOVA Research Journal, vol. 5, n ${ }^{\circ}$ 3.1, pp. 76-88. https://doi.org/10.33890/innova.v5.n3.1.2020.1491

[39] Fondo de las Naciones Unidas para la Infancia, "La comprensión lectora", 2010.

[40] Organización de las Naciones Unidas para la Educación, la Ciencia y la Cultura, "Participación de las familias en la educación infantil latinoamericana". Santiago de Chile: Oficina Regional para la Educación de América Latina y el Caribe, 2014.

[41] Organization for Economic Cooperation and Development, "PISA for Development Assessment and Analytical Framework: Reading, Mathematics and Science", París: OECD Publishing, 2017.

[42] Ministerio de Educación Nacional, “Cartilla para Padres de Familia. ¿Cómo participar en los procesos educativos de la escuela?”, 2007.

[43] Ministerio de Educación Nacional, "Plan Nacional de Lectura y Escritura (PNLE)", 2016.

[44] M. Velazco y F. Mosquera, "Estrategias didácticas para el aprendizaje colaborativo", 2010.

[45] T. Izquierdo, M. Sánchez y M. López, "Determinantes del entorno familiar en el fomento del hábito lector del alumnado de Educación Primaria”. Estudios sobre Educación, vol. 36, n 1, pp. 157-179, 2019. https://doi.org/10.15581/004.36.157-179

[46] I. Solé, "Competencia lectora y aprendizaje”. Revista iberoamericana de educación. Didáctica de la Lengua y la Literatura, vol. 59, n 1 , pp. 43-61, 2011. https://doi.org/10.35362/rie590456

[47] C. Mekis, "El Centro de Recursos para el Aprendizaje, CRA. La nueva biblioteca multimedial que responde a las transformaciones y desafíos de la nueva educación", España: UNESCO / CERLALC, 2007.

[48] D. Cassany, "Los enfoques comunicativos: Elogio y crítica". Lingüística y Literatura, vol. 36-37, n 1, pp. 11-33, 1999.

[49] A. Maslow, "El hombre autorrealizado, hacia una psicología del ser", Buenos Aires: Troquel, 1968.

[50] M. Petit, "La lectura, íntima y compartida", 2017.

[51] Ministerio de Educación Nacional, "Familias lectoras. Cómo formar lectores y escritores en el hogar", Bogotá: Serie Río de Letras, Manuales y Cartillas PNLE, 2016.

[52] Fundación Jaume Bofill, "LECXIT, Lectura para el éxito educativo", 2017.

[53] Instituto Colombiano para la Evaluación de la Educación, "Guía de uso e interpretación de resultados”, Bogotá: Ministerio de Educación Nacional, 2018. 